Dear Editor,

\title{
Asymptotics of the Luria-Delbrück distribution
}

The letter Pakes (1993) and the note Kemp (1994) both include asymptotic evaluations of the probabilities of the Luria-Delbrück distribution, which is used in models for bacteria acquiring resistance to a lethal virus by mutation. The asymptotic behaviour is highly relevant to certain arguments about the nature of the mutation process (cf. Cairns et al. (1988), Kemp (1994)). The letter employs the theory of subexponential probability distributions while the note uses direct calculation. The purpose of the present letter is to point out a third, very simple, method of obtaining the asymptotics, using regularly varying sequences. This is related to the subexponential approach but slightly more direct. It avoids the calculation of the convolution of a Luria-Delbrück distribution with itself that is inherent in the subexponential approach. I hope that by bringing together Pakes (1993) and Kemp (1994) with the approach below, I will give the reader a useful overview of these various sorts of asymptotic techniques.

The Luria-Delbrück distribution $\mathrm{LD}(m)$, with a positive parameter $m$, is that of a non-negative integer-valued random variable $Y$, say:

$$
\boldsymbol{P}(Y=n)=p_{n} \quad(n=0,1,2, \cdots),
$$

where $Y$ is built as a Poisson compound, as follows. Let $N, X_{1}, X_{2}, \cdots$ be mutually independent, with $N$ being Poisson-distributed with mean $m$, while each $X_{i}$ has the distribution

$$
\boldsymbol{P}\left(X_{i}=n\right)=\alpha_{n}:=\frac{1}{n}-\frac{1}{n+1} \quad(n=1,2, \cdots)
$$

Then put $Y:=\sum_{n=1}^{N} X_{n}$, where, as is the usual convention, $\Sigma_{n=1}^{0}$ is interpreted as 0 .

The probability generating function (p.g.f.) of the $\operatorname{LD}(m)$ distribution, $g(z)=\boldsymbol{E} z^{Y}=$ $\sum_{n=0}^{\infty} p_{n} z^{n}$, turns out to be given by

$$
g(z)=(1-z)^{m(1-z) / z} \quad(|z| \leqq 1) .
$$

To see this, note that the compound Poisson construction of $Y$ implies that 


$$
g(z)=e^{-m(1-h(z))} \quad(|z| \leqq 1),
$$

where $h$ is the p.g.f. of the common distribution $\left(\alpha_{n}\right)$ of the $X_{i}$ :

$$
h(z)=\sum_{n=1}^{\infty} \alpha_{n} z^{n}=1+\left(\frac{1}{z}-1\right) \ln (1-z) \quad(|z| \leqq 1) .
$$

Substitution of (3) into (2) yields (1).

Now (2) yields the identity of $g^{\prime}(z)=m h^{\prime}(z) g(z)$, which expands to give the recursive relations

$$
n p_{n}=m \sum_{j=1}^{n} j \alpha_{j} p_{n-j} \quad(n=1,2, \cdots), \quad \dot{p}_{0}=e^{-m},
$$

for the $p_{n}$ in terms of the $\alpha_{n}$. And (4) allows a simple general result linking the asymptotics of $p_{n}$ and $\alpha_{n}$ to be applied: Theorem 3.1 of Hawkes and Jenkins (1978). For, putting $b_{n}$ : $=e^{m} p_{n}$ and $a_{n}:=m n \alpha_{n}$, we have $b_{0}=1, a_{n} \geqq 0$, and $n b_{n}=\sum_{j=1}^{n} a_{j} b_{n-j}$ for $n \geqq 1$. The sequence $\left(a_{n}\right)$ is regularly varying of index -1 , that is, with $\lfloor\cdot\rfloor$ denoting integer part, $a_{\left\lfloor\lambda_{n}\right\rfloor} / a_{n} \rightarrow \lambda^{-1}$ as $n \rightarrow \infty$, for each fixed $\lambda>1$. Under these conditions the Hawkes-Jenkins theorem gives $b_{n} \sim B a_{n} / n$ as $n \rightarrow \infty$, where $B:=\Sigma_{0}^{\infty} b_{n}=e^{m}$. (By the notation $u_{n} \sim v_{n}$ is meant that $u_{n} /$ $v_{n} \rightarrow 1$.) It follows that $p_{n} \sim m \alpha_{n}$, which re-establishes the conclusion at the end of Kemp (1994), equivalent to (9) of Pakes (1993), that

$$
\lim _{n \rightarrow \infty} n(n+1) p_{n}=m \text {. }
$$

As Kemp observes, (5) implies immediately that $P_{n}:=\sum_{j=n}^{\infty} p_{j} \sim m / n$ as $n \rightarrow \infty$, and 'This establishes mathematically the feature of the Luria-Delbrück distribution that was so important in Cairns et al. (1988).'

\section{Acknowledgment}

I thank the referee for bringing Pakes (1993) to my attention.

\section{References}

Cairns, J., Overbaugh, J. AND Miller, S. (1988) The origin of mutants. Nature 335, 142-145. HaWkes; J. AND Jenkins, J. D. (1978) Infinitely divisible sequences. Scand. Actuarial J., 65-76. Kemp, A. W. (1994) Comments on the Luria-Delbrück distribution. J. Appl. Prob. 31, 822-828. Pakes, A. G. (1993) Remarks on the Luria-Delbrück distribution. J. Appl. Prob. 30, 991-994.

School of Mathematical Sciences Yours sincerely Queen Mary and Westfield College Charles M. Goldie (University of London)

Mile End Road

London E1 4NS, UK 